\title{
CLINICAL PRACTICE The dilemma of treating major burns in South Africa
}

\author{
H Rode, A Rogers, S Adams, W Kleintjes, L Whitelock-Jones, A Muganza, N Allorto \\ The authors represent the South African Burn Society. H Rode and A Rogers are with the Red Cross War Memorial Children's Hospital and the \\ University of Cape Town, South Africa. S Adams is with Groote Schuur Hospital and the University of Cape Town, South Africa. W Kleintjes is \\ with Tygerberg Hospital and the University of Stellenbosch. L Whitelock-Jones is past president of the South African Burn Society. A Muganza \\ is with the Johnson and Johnson Burns Unit, Baragwaneth Hospital and University of the Witwatersrand and is President of the South African \\ Burn Society. N Allorto is the secretary/treasurer of the association.
}

Corresponding author: H Rode (heinz.rode@uct.ac.za)

The major challenge facing South African burn surgeons is the current and future management of major burns. Survival is greatly dependent on adequate therapeutic measures. Various therapeutic measures can be instituted to overcome the shortage of donor skin, as we describe. Each of these methods has its specific role in the management of major burns. The use of very expensive and highly specialised techniques for individual patients cannot be justified when more accessible and cost-effective methods are available. To serve the greater population of burn victims, every effort should be made to use these available methods rather than channelling scarce financial resources into procedures that have a very limited role in South Africa.

S Afr Med J 2013;103(9):608-609. DOI:10.7196/SAMJ.7361

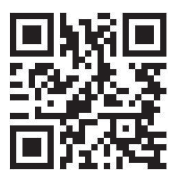

The South African Medical Research Council has estimated that approximately $3.2 \%$ of the South African (SA) population suffer from thermal injuries annually. ${ }^{[1]}$ The vast majority of these victims are from poorer communities and only $6 \%$ of patients will consult the private sector, while the greater majority are cared for by various provincial facilities. It is estimated that less than R4 000 on average is spent on a burns patient per year. Compounding management further is that burn care in SA is variable in terms of organisation, management, facilities, staffing, clinical workload and outcomes.

The major challenge facing SA burn surgeons is the current and future management of major burns. A major burn is defined as one exceeding $30 \%$ of the total body surface area (TBSA). Survival is greatly dependent on adequate therapeutic measures, including resuscitation, infection control, early wound closure, management of inhalation injury, adequate nutrition, pain control and acute rehabilitation.

After sustaining a deep partial or full thickness burn, removal of the dead tissue within 2 - 5 days is the accepted stan 'dard of care. However, in burns exceeding 50\% TBSA there is insufficient nonburn tissue (donor site) to provide sufficient autografts for early definitive closure. Fifteen percent of the surface area of the patient cannot be used as donor site. To overcome the shortage of donor skin various therapeutic measures can be instituted:

1. The patient's harvested skin can be widely meshed from 1:3 to 1:6, thereby expanding the skin to cover a greater area. This technique, however, requires expanded grafts to be covered with either allografts or a synthetic substitute like Biobrane to prevent desiccation of the interstitial areas. Results are satisfactory but leave a corrugated skin pattern on healing.

2. In the absence of sufficient donor skin, an alternative method to cover the excised burn wound, is the MEEK system. This is based on a centuries-old Chinese technique whereby the donor skin is divided into multiple small pieces (micrografts), consisting of both epithelial and dermal tissue, and then placed onto the recipient area. The development of the MEEK system has significantly altered the management of major burns by enabling surgeons to expand skin between 1:3 and 1:9. This technique is ideally suited for large burns with limited donor sites.
3. For temporary wound closure, cadaveric skin or allografts are the 'gold standard'. This is a very feasible, satisfactory and inexpensive method. Because the cadaveric skin functions as a temporary biological dressing, there is no need for tissue typing. The cadaveric skin promotes epithelialisation, suppresses infection, prepares the wound bed for definitive closure, provides a dermal template for epithelial grafts and prevents desiccation of tissue. Allografts can either be used immediately after procurement or cryopreserved for later use. Allografts used to be freely available from both private and state funeral parlours and from solid organ donors. Unfortunately, SA regulations changed a few years ago: whereas previously duty police officers were permitted to obtain consent from the deceased's family for allograft procurement, the new regulations prescribe that only a forensic pathologist may legally do so. This has resulted in a remarkable reduction in the availability of allografts for burn care. This situation is unacceptable and there is an urgent call for the establishment of a skin bank in SA. An additional impetus for establishing such a skin bank would be the necessity of being able to handle a mass disaster, where it is estimated that more than $25 \%$ of victims may suffer from burn wounds. In such circumstances, xenografts could be used, but sterility, availability and costs may limit their use.

4. In verylarge life-threatening burns, cultured epithelial autografts (CEA) represent an additional and potentially lifesaving method achieving epithelial cover. There are two techniques: the so-called 'spray-on skin' and 'sheet grafts'. Both these methods require procurement of a small $1 \mathrm{~cm}^{2}$ skin biopsy. In the spray-on skin technique the biopsy is sub-cultured in growth medium for 5 days and the suspension is then sprayed onto the recipient area. These cells are non-confluent in comparison to the sheet autograft and, once deposited, multiply rapidly. The recipient area is covered within 5 days with new growth of epithelial tissue on a dermal base: $1 \mathrm{~cm}^{2}$ can expand to cover 500 $\mathrm{cm}^{2}$. The second method of growing epithelial cells on a template is controversial. The sheet graft consists only of epithelial cells which are in a confluent state and therefore do not multiply. The production of these sheet grafts is hugely expensive. They should be used only as an adjunct to conventional autografting in large burns and not as a 
method of improving the cosmetic appearance of minor to moderate size burns. The resulting epidermis remains very fragile with frequent blistering and contractures. There is no dermal component or UV protection and at least six children in the United States (US) have developed carcinoma with these grafts (Personal communication, Dr Nathan Kenalyam, Head of Department of the Burns Unit, Oregon Health and Science University, School of Medicine, Oregon, Portland, US). It takes 3 - 4 weeks to grow sufficient cells from a tissue biopsy to resurface the wound. This long delay compromises the patient. Such grafts must be used on delivery. Successful engraftment varies from $50 \%$ to $75 \%$ and is best done in the acute phase. At present, most US surgeons believe that CEA should be reserved for the treatment of massive burns when other methods, outlined above, have failed or cannot be used. ${ }^{[2]}$ The use of CEA must therefore be seen as a rescue method in very large burns. Most surgeons today will use this type of graft in conjunction with a bioengineered dermis or allografts from which the epidermis has been removed, to improve the results.

5. To facilitate permanent wound cover, there are a number of commercially available products: Alloderm is an acellular human dermal allograft (and must eventually be covered by autologous autograft). Other bio-engineered dermal regenerative templates to create a vascularised collagenous dermal layer are Integra and Pelnac. Once the neodermis has been vascularised, an epidermal autograft is applied. These techniques provide a permanent composite skin.

We are of the opinion that each of these various methods has its specific role in the management of major burns. The use of very expensive and highly specialised techniques for individual patients cannot be justified against more accessible and cost-effective methods for a large number of patients.

To serve the greater population of burn victims, every effort should be made to establish a skin bank for universal use, rather than channelling scarce financial resources into procedures that have a very limited role in SA.

\section{References}

1. Matzopoulos RE. A Profile of Fatal Injuries in South Africa. Fifth Annual Report of the National Injury Mortality Surveillance System. Cape Town: University of Cape Town and the MRC Crime, Violence and Injury Lead Program, 2004

2. Shores I Gabriel A Gupta S. Skin substitutes and alternatives: A review. Advances Skin \& Wound Care 2007;20(9):493-508. [http://dx.doi.org/10.1097/01.ASW.0000288217.83128.f3] 